Annuaire suisse de politique de développement

27-2 | 2008

Migration et développement: un mariage arrangé

\title{
Une revue historique et sociologique des migrations en Suisse
}

Gianni D'Amato

\section{(2) OpenEdition}

12 Journals

Édition électronique

URL : http://journals.openedition.org/aspd/511

DOI : 10.4000/aspd.511

ISSN : 1663-9669

Éditeur

Institut de hautes études internationales et du développement

\section{Édition imprimée}

Date de publication : 1 décembre 2008

Pagination : 169-187

ISBN : 978-2-940415-07-6

ISSN : 1660-5934

\section{Référence électronique}

Gianni D'Amato, « Une revue historique et sociologique des migrations en Suisse », Annuaire suisse de politique de développement [En ligne], 27-2 | 2008, mis en ligne le 22 mars 2010, consulté le 08

septembre 2020. URL : http://journals.openedition.org/aspd/511; DOI : https://doi.org/10.4000/aspd. 511

(C) The Graduate Institute I Geneva 


\title{
Une revue historique et sociologique des migrations en Suisse
}

\author{
Gianni D'Amato*
}

\section{Considérations "tardives" de la recherche \\ (et de la pratique) sur la réalité des migrations}

Un bilan du phénomène migratoire en Suisse, aussi incomplet qu'il apparaisse ici, offre néanmoins l'occasion de remettre en question des critères figés de la recherche sociologique sur les migrations et de considérer d'un autre point de vue, avec plus de recul surtout, un domaine politique où des interventions s'imposent de toute urgence. Avec la pression qui pèse sur la gestion de la recherche universitaire, force est de constater que les institutions académiques spécialisées ne peuvent que réagir à l'évolution du débat sur les migrations et de la politique migratoire, au lieu de dégager des ressources pour la recherche à long terme qui permettraient de garantir une étude interdisciplinaire et suivie de l'immigration, qui est une composante constitutive de toute société moderne.

Or, malgré toute l'actualité politique et thématique du phénomène, la recherche sur les migrations a omis de saisir sa complexité et d'analyser les intérêts opposés des acteurs en présence - les migrants, de même que les pays d'immigration et d'émigration - de telle sorte que les résultats de son analyse puissent servir à élaborer une politique d'intégration digne de ce nom ${ }^{1}$. A ce manquement du côté de la science fait pendant la négligence de la politique. Dans nombre de pays européens, celle-ci s'est en effet montrée incapable, ces trente à quarante dernières années, de comprendre à temps que la «main-d'œuvre étrangère» était devenue une minorité immigrée et de se donner ainsi les moyens de concevoir des politiques démographiques applicables à long terme. Notre propos est d'esquisser un aperçu critique des principaux aspects des migrations en Suisse et de fournir quelques points de repère permettant d'appréhender les migrations et le développement dans le cadre des profonds changements sociaux intervenant à l'échelle mondiale.

\section{La Suisse, pays d'immigration?}

Au fil des siècles, la Suisse s'est fait connaître pour sa neutralité, sa stabilité économique et sociale, sa diversité linguistique et culturelle et une combinaison unique de démocratie directe et de fédéralisme. Depuis le début des temps modernes, elle s'est ainsi forgé la réputation de gardienne du libéralisme en

* Professeur et directeur, Forum suisse pour l'étude des migrations et de la population (SFM), Université de Neuchâtel.

1 Pour une première argumentation dans ce sens, voir Kingsley Davis, «Social Science Approaches to International Migration», Population and Development Review, vol. 14, nº 4, 1988, p. 245. 
Europe, ce qui a exercé un attrait certain sur les victimes de persécutions, mais aussi sur d'autres personnes à la recherche d'un nouveau foyer économique, spirituel et politique. Les huguenots et d'autres réfugiés protestants furent parmi les premiers à y trouver asile au XVI ${ }^{e}$ et au XVII ${ }^{e}$ siècle, après le massacre de la Saint-Barthelémy et la guerre de Trente Ans. Au XIX ${ }^{\mathrm{e}}$ siècle, divers exilés européens profitèrent également du triomphe du libéralisme suisse. C'est surtout après l'échec des insurrections de mars 1848 qu'ils vinrent se réfugier au sein de la Confédération ${ }^{2}$.

Il a fallu attendre la seconde moitié du XIX ${ }^{\mathrm{e}}$ siècle et l'accélération de l'industrialisation pour voir la Suisse se muer en un pays d'immigration de type moderne. Contrairement à l'image d'Epinal qu'on lui associait alors - celle d'un pays alpin essentiellement campagnard -, la Suisse fit, à cette époque déjà, partie de l'avantgarde européenne dans divers secteurs d'activités: tout d'abord l'industrie textile, puis l'industrie des machines et l'industrie chimique. Pour jouer ce rôle de pionnière, elle a dû consentir d'importants investissements dans l'industrie et les infrastructures, mais aussi dans l'acquisition et la diffusion de connaissances universitaires. En parallèle, le secteur agricole s'est contracté et nombre des personnes qu'il occupait ont soit rejoint les rangs de l'industrie, soit quitté la Suisse pour reconstruire leur existence outre-mer ${ }^{3}$. L'immigration a commencé dès le milieu du XIX ${ }^{e}$ siècle. Elle fut notamment marquée par l'arrivée d'universitaires, d'artisans et de membres de professions libérales venus d'Allemagne, mais aussi d'Italie, qui trouvèrent du travail dans la science, l'industrie, la construction et la réalisation d'infrastructures ${ }^{4}$. Leurs rangs ont rapidement gonflé, surtout de 1888 à 1914: entre 1888 et 1919, le nombre des Allemands présents en Suisse a ainsi doublé pour passer de 112000 à 220000 , tandis que la communauté italienne est passée de 117000 à 203000 membres entre 1900 et $1910^{5}$.

Au cours de cette première phase d'immigration, soit avant la Première Guerre mondiale, la responsabilité de la politique migratoire incombait aux cantons, leur législation devant toutefois respecter les conditions des accords bilatéraux conclus par la Suisse avec d'autres Etats pour réguler l'immigration. A l'instar d'autres traités de l'époque portant sur la libre circulation des personnes, ces accords ne s'opposaient pas à l'immigration, car ils visaient aussi à garantir aux Confédérés la possibilité d'émigrer. Après une première campagne politique, menée vers la fin de la Première Guerre mondiale déjà, contre les étrangers

2 Thomas Busset, «La politique du refuge en Suisse 1820-1870. Réalité et mythe», Zeitschrift des Schweizerischen Bundesarchivs, $\mathrm{n}^{\circ}$ 25, 1999, p. 34.

3 Dieter Freiburghaus und Brigitte Guggisberg, «Die schweizerische Ausländerpolitik seit 1850: eine Analyse auf dem Hintergrund politisch-ökonomischer Paradigmen », in Thomas Geiser, Hans Schmid und Emil Walter-Busch (Hrsg.), Arbeit in der Schweiz des 20. Jahrhunderts: wirtschaftliche, rechtliche und soziale Perspektiven, Berne (etc.), P. Haupt, 1998, pp. 137-185.

4 Une étude de Klaus Urner explore en détail l'hétérogénéité de l'immigration allemande au XIX ${ }^{e}$ siècle, de même que son rôle dans le développement de la vie économique, culturelle et politique en Suisse avant la Première Guerre mondiale (Klaus Urner, Die Deutschen in der Schweiz: von den Anfängen der Kolonienbildung bis zum Ausbruch des Ersten Weltkrieges, Frauenfeld (etc.), Huber, 1976). Pour ce qui est des Italiens en Suisse, voir les publications de Marc Vuilleumier: «L'emigrazione italiana in Svizzera e gli avvenimenti del 1898» (in AA. VV., Anna Kuliscioff e l'età del riformismo : atti del convegno di Milano, dicembre 1976, Roma, Mondo operaio, 1978, pp. 85-103) et «Le mouvement ouvrier et les travailleurs italiens en Suisse jusqu'à la Première Guerre mondiale» (Revue syndicale suisse, vol. 82, $\mathrm{n}^{\circ} 4,1990$, pp. 102-116).

5 Voir Madelyn Holmes, Forgotten Migrants: Foreign Workers in Switzerland before World War I, Rutherford (etc.), Fairleigh Dickinson University Press (etc.), 1998, p. 15. 
vivant en Suisse, la population a accepté en 1925 d'inscrire dans la Constitution un article qui confère au Conseil fédéral le pouvoir de régir l'établissement des étrangères et des étrangers à l'échelon fédéral. Cet article servit aussi de base légale à la création de la police des étrangers et à l'élaboration de la loi fédérale sur le séjour et l'établissement des étrangers (LSEE), adoptée en 1931. Dès ce moment-là, l'immigration devait respecter les intérêts moraux et économiques du pays et tenir compte du degré de «surpopulation étrangère». Pendant des décennies, le consensus politique qui régna autour de la xénophobie culturelle et démographique a empêché la Suisse, jusqu'à l'entrée en vigueur en 2008 de la nouvelle loi sur les étrangers (LEtr), de mener une politique d'immigration cohérente, axée sur l'intégration des étrangers. Une fois leur travail accompli, les travailleuses et les travailleurs immigrés étaient censés quitter le pays.

\section{Les migrations de main-d'œuvre après la Seconde Guerre mondiale}

Après la Seconde Guerre mondiale, on a assisté à une nouvelle promotion de l'émigration, car les pays occidentaux devaient stabiliser leur économie dans le système de marché régi par les institutions de Bretton Woods. Escomptant une relance conjoncturelle, la Suisse a signé un accord de recrutement de maind'œuvre avec l'Italie en 1948 déjà. La majeure partie de cette main-d'œuvre italienne fut employée dans le secteur des infrastructures et de la construction, ainsi que dans l'industrie des machines et du textile.

En édictant une législation sur les questions migratoires (LSEE), l'Etat s'était doté de procédures régissant l'accès au marché suisse du travail. Relativement souple, le système de l'établissement prévoyait divers types d'autorisations autorisation de séjour saisonnière (permis A), autorisation de séjour annuelle (permis B), autorisation d'établissement (permis C), autorisation de courte durée (permis L) et autorisation pour demandeurs d'asile (permis N) - sur lesquels nous reviendrons plus en détail par la suite. Dans ce sens, la politique migratoire peut être considérée comme une tentative ciblée d'influer sur les flux migratoires au moyen d'instruments étatiques. C'est en particulier la politique d'admission qui subissait sans cesse le feu croisé de la critique. Pour s'assurer que les migrantes et les migrants n'allaient pas s'établir définitivement en Suisse, dès 1945 on a augmenté de cinq à dix ans la durée de séjour donnant droit à un tel établissement, tout en restreignant les conditions du regroupement familial. La politique misait alors sur la rotation de la main-d'œuvre étrangère, c'est-à-dire sur le remplacement régulier des migrants présents par de nouveaux travailleurs, pour que les premiers ne puissent pas s'établir et ne contribuent pas à la «surpopulation étrangère» du pays. Voilà pourquoi les travailleuses et les travailleurs ne se voyaient octroyer, au début de leur séjour, qu'un permis de travail d'une année, qui pouvait cependant être prolongé, et l'était souvent.

A la suite de la réactivation de la politique de recrutement, le nombre des immigrants définitivement établis en Suisse est passé en dix ans de 271000 en 1950 à 476000 en 1960 , pour grimper à 1080000 en $1970^{6}$. Si cette main-

6 Hans Mahnig et Etienne Piguet, «La politique d'immigration suisse de 1948 à 1998: évolution et effets », in Hans-Rudolf Wicker, Rosita Fibbi et Werner Haug (dir.), Les migrations et la politique suisse. Résultats du Programme national «Migrations et relations interculturelles », Zurich, Seismo, 2003, pp. 63-103. 
d'œuvre provenait au début essentiellement d'Italie, les régions de provenance se sont diversifiées dès les années 1960: en 1970, plus de la moitié des étrangères et des étrangers sont encore arrivés d'Italie, mais $20 \%$ sont venus d'Allemagne, de France et d'Autriche, $10 \%$ d'Espagne et $4 \%$ de Yougoslavie, du Portugal et de Turquie.

La conjoncture économique se maintenant au beau fixe dans les années 1960, tant l'économie que la politique ont progressivement remisé le principe de rotation au second plan. Par ailleurs, la demande croissante de main-d'œuvre à travers toute l'Europe et les pressions politiques des pays d'origine ont contribué à améliorer les conditions de séjour des étrangers en Suisse. Dans ce contexte, force était à la diplomatie économique suisse de faire des concessions ${ }^{7}$. Dans le sillage du deuxième accord bilatéral de recrutement signé avec l'Italie en 1964, les autorités suisses ont tenté de remplacer le système de rotation par un modèle axé sur l'intégration des étrangers. Dès les années 1970, les représentants des migrants ont pour leur part aussi commencé à parler de l'intégration des travailleurs étrangers, comprise comme la reconnaissance de l'égalité de leurs droits, tant sociaux qu'économiques et politiques. Leurs principales revendications comprenaient une meilleure défense de leurs intérêts auprès des syndicats, le regroupement familial, la suppression des classes spéciales à l'école et d'autres formes d'inégalités dans l'enseignement. Formulées ouvertement par les organisations de migrants, ces prétentions ont toutefois apporté de l'eau au moulin du mouvement xénophobe, qui voulait pour sa part réduire la proportion d'étrangers au sein de la population suisse et rejetait toute velléité visant à étendre leurs droits sociaux. En matière d'intégration, les syndicats étaient divisés: alors que les uns comprenaient la nécessité d'intégrer la main-d'œuvre étrangère dans leurs rangs, d'autres - surtout parmi les dirigeants syndicaux ne considéraient les «travailleurs étrangers» que comme des concurrents sur le marché de l'emploi ${ }^{8}$.

Après le choc pétrolier de 1973 et le fléchissement conjoncturel qui s'ensuivit, nombre de travailleurs étrangers durent quitter le pays, car beaucoup ne disposaient pas d'une assurance chômage. La Suisse a ainsi pu exporter le chômage vers les pays d'origine en limitant l'octroi de permis de séjour. A l'époque, la part de la population étrangère a diminué de 17,2\% à 14,8\%. La nouvelle maind'œuvre étrangère, arrivée avec le redressement de la situation économique vers la fin des années 1970, ne provenait plus seulement d'Italie, mais de plus en plus d'Espagne, du Portugal et de Turquie. La part des immigrés dans la population s'est alors à nouveau accrue, passant de $14,8 \%$ en 1980 à $16,4 \%$ en 1990 , puis à 19,5\% en 2000 (voir graphique 1).

7 Mauro Cerutti, «L'immigration italienne en Suisse dans le contexte de la Guerre froide», in Jean Batou, Mauro Cerutti et Charles Heimberg (dir.), Pour une histoire des gens sans histoire: ouvriers, exclus et rebelles en Suisse: XIX $X^{e}-X X^{e}$ siècles, Lausanne, Editions d'En Bas, 1995, pp. 213-231.

8 Jean-Pierre Fragnière et Roger Girod (dir.), Dictionnaire suisse de politique sociale, Lausanne, Réalités Sociales, 2002. 
Graphique 1: Part des étrangers dans la population résidante, 1860-2007 (en pourcentage)

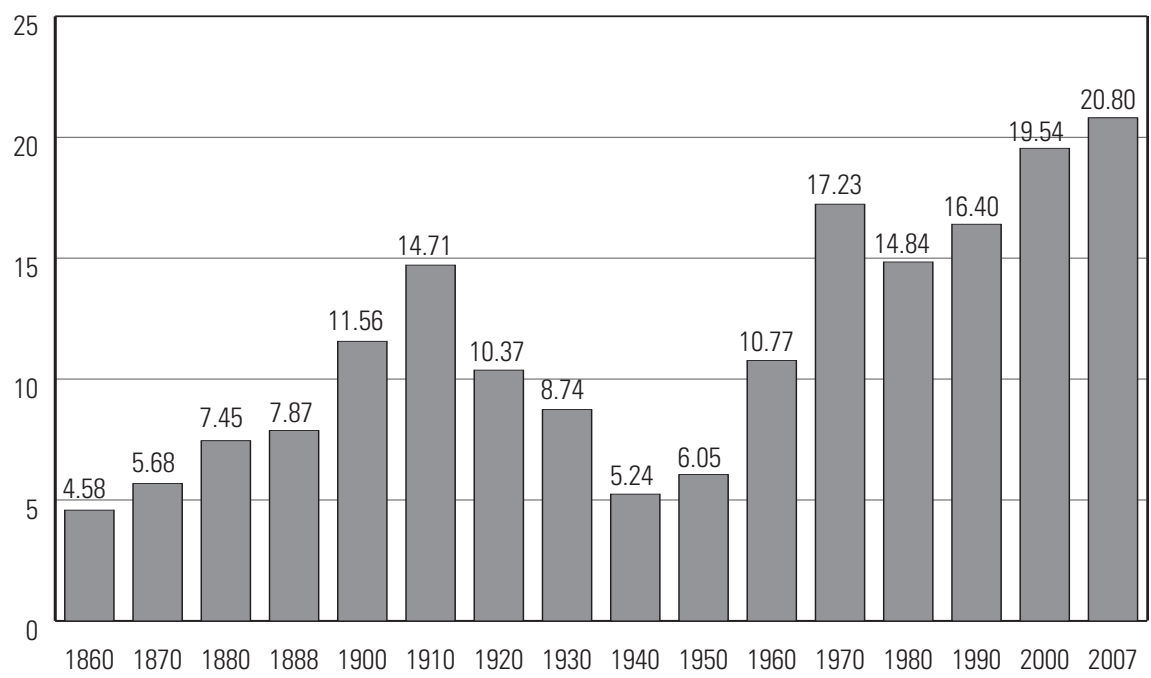

Sources: Office fédéral de la statistique (OFS) (dir.), Annuaire statistique de la Suisse 2008, Neuchâtel, OFS, 2008. Office fédéral des migrations (ODM), "Statistique des étrangers», 2007.

La crise économique des années 1990 a à nouveau frappé de plein fouet la main-d'œuvre étrangère non qualifiée et à présent plus âgée. Le chômage a atteint des sommets parmi ces travailleurs qui peinaient, faute de qualifications appropriées, à se réinsérer dans le monde du travail. La Suisse a ainsi connu un taux de chômage structurel inégalé depuis la Seconde Guerre mondiale. Obligées d'assumer les conséquences financières et sociales de cette crise, plusieurs villes ont demandé à la Confédération de s'engager davantage à leurs côtés et obtinrent gain de cause. A la suite de l'entrée en vigueur, en 1999, de l'article sur l'intégration, la Confédération a pour la première fois pris part à la promotion de l'intégration par les communes ${ }^{9}$. Le rôle de l'intégration acquit ainsi une nouvelle dimension. Outre la reconnaissance mutuelle, il fut pour la première fois question de créer de nouvelles compétences, tant au sein des communautés immigrées que de la population suisse ${ }^{10}$, tous les segments de la population devant à l'avenir pouvoir se comprendre aisément. Les principaux objectifs étaient d'accroître les compétences linguistiques et les échanges, ainsi que de supprimer les barrières limitant l'accès à l'éducation, à la santé et aux autorités. La nouvelle Loi fédérale sur les étrangers (LEtr), entrée en vigueur en 2008 pour remplacer la LSEE de 1931, comprend désormais un chapitre consacré à l'intégration. On peut toutefois l'interpréter comme une exigence adressée aux étrangers: s'intégrer dans la société ou s'en aller. La nouvelle loi met pour la première fois l'accent sur les intérêts de toute l'économie, et non plus seulement sur ceux de certains secteurs d'activités, et vise plus particulièrement l'immigration de main-d'œuvre non européenne hautement qualifiée ou celle de ressortissants de pays de l'Association européenne de libre-échange (AELE) et de

9 Gianni D'Amato und Brigitta Gerber (Hrsg.), Herausforderung Integration: städtische Migrationspolitik in der Schweiz und in Europa, Zurich, Seismo, 2005.

10 Jean-Pierre Fragnière et Roger Girod, op. cit. 
l'Union européenne (UE), dont l'admission est régie par les accords bilatéraux avec l'UE en vigueur depuis 2002. Elle octroie également aux étrangers au bénéfice d'une autorisation de séjour une plus grande liberté sur le marché suisse du travail et le droit, sans délai préalable, au regroupement familial.

Les statistiques illustrent la vitesse à laquelle la législation s'adapte aux nouveaux besoins de l'économie et à laquelle les flux migratoires réagissent à un changement du contexte général. En effet, si quelque 300000 travailleurs étrangers ( $46 \%$ de la main-d'œuvre étrangère) étaient employés dans l'industrie en 1970, à l'ère de la haute conjoncture, ils n'étaient plus que 169000 (ou 20,4\%) en $2005^{11}$. Depuis lors, la part de la main-d'œuvre a également suivi une baisse continue dans la construction, mais a légèrement augmenté dans l'hôtellerie entre 1970 et 2005. Le recul de ces trois branches, qui employaient traditionnellement la main-d'œuvre étrangère depuis la Seconde Guerre mondiale, est à mettre sur le compte de l'essor du secteur des services. Alors que les sociétés de crédit, d'immobilier et d'assurances n'employaient que 18000 personnes de nationalité étrangère en 1970 (soit 2,7\% de l'ensemble de la main-d'œuvre étrangère), leur nombre était passé à 133000 en 2005 (ou 16,1\%). Ce changement structurel se retrouve aussi dans la diversification des régions de provenance des étrangers: depuis le début de ce siècle et l'entrée en vigueur des accords bilatéraux, le nombre des immigrés venant d'Allemagne et du Portugal ne cesse de croître, tandis que les ressortissants des pays traditionnels de recrutement (Italie, Espagne et ancienne Yougoslavie) se font sans cesse moins nombreux. Cette évolution s'explique non seulement par le retour des migrantes et des migrants plus âgés dans leur pays, mais aussi par la tendance à la naturalisation parmi les «anciens» immigrants. Si la plupart des immigrés allemands correspondent au profil de qualification requis par le secteur des services, les Portugais occupent surtout le secteur des bas salaires, qui reste un grand demandeur de main-d'œuvre ${ }^{12}$. En 2007, les étrangères et les étrangers venus en Suisse pour y exercer une activité lucrative représentaient déjà $44,4 \%$ de l'immigration annuelle (contre $35 \%$ en 2004). Nombre d'autres ressortissants étrangers sont par ailleurs entrés en Suisse au titre du regroupement familial (32,3\% en 2007 et $40,3 \%$ en 2004). Enfin, les personnes qui viennent suivre une formation ou un perfectionnement professionnel forment un autre gros segment de la population étrangère (10,5\%) (voir graphique 2$)$.

En Suisse, la notion de migrantes et de migrants englobe le plus souvent aussi les membres de la deuxième génération, c'est-à-dire les jeunes étrangers nés ou ayant grandi en Suisse, sans être eux-mêmes des immigrants. Alors que des études antérieures présentent leur situation comme le résultat de conditions génératrices d'anomie, des recherches plus récentes ont corrigé cette image ${ }^{13}$. Si on leur ajoute les personnes naturalisées, les jeunes d'origine étrangère font figure d'overperformers face à leurs contemporains suisses issus du même

11 Bien entendu, l'industrie de transformation employait également de la main-d'œuvre suisse: 741775 personnes (ou 31,8\%) en 1970, 476000 (ou 15,1\%) en 2005. Ces données sont tirées de l'ouvrage d'Etienne Piguet, Einwanderungsland Schweiz: fünf Jahrzehnte halb geöffnete Grenzen, Bern (etc.), Haupt Verlag, 2006.

12 Voir Philippe Wanner, Migration et intégration: Populations étrangères en Suisse, Neuchâtel, Office fédéral de la statistique, 2004, p. 61.

13 Claudio Bolzman, Rosita Fibbi et Marie Vial, Secondas - secondos: le processus d'intégration des jeunes adultes issus de la migration espagnole et italienne en Suisse, Zurich, Seismo, 2003. 
milieu social, en particulier pour ce qui est de la mobilité sociale. Quoique parfaitement intégrés dans les structures suisses, ils parviennent à maintenir un équilibre étonnant entre le monde de leur famille et la culture de leur environnement suisse. La découverte de cette «assimilation segmentée» (Alejandro Portes) a été confirmée et précisée par une étude de l'Office fédéral de la statistique: la réussite relative des membres de la deuxième génération a été constatée chez tous les jeunes d'origine étrangère nés en Suisse, quels que soient le moment de l'immigration et leur statut légal (étrangers ou suisses). La possession de la nationalité suisse n'est cependant d'aucun secours aux jeunes d'origine étrangère aux qualifications insuffisantes, en particulier s'ils sont nés à l'étranger ${ }^{14}$.

\section{Graphique 2: Etrangers entrés en Suisse par motif d'immigration, 2007 (en pourcentage)}

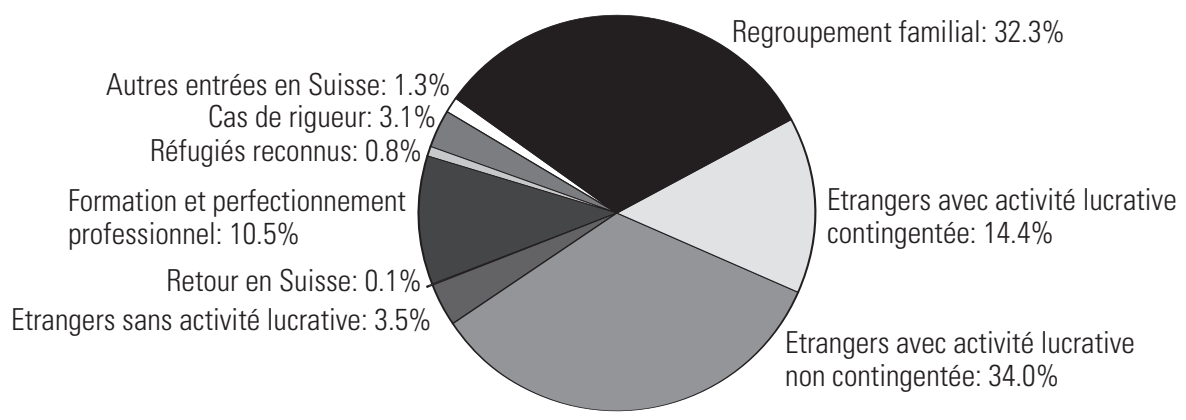

Source: Office fédéral des migrations (ODM), "Statistique des étrangers», 2007

Le changement de la législation a dans l'ensemble exercé une influence sur le type de permis de séjour de la population résidante étrangère. L'immense majorité des membres des communautés italienne, espagnole et portugaise bénéficie ainsi d'une autorisation d'établissement (94\% des Italiens, 93\% des Espagnols et $83 \%$ des Portugais ont un permis C), tandis que moins de $40 \%$ des Africains, des Asiatiques et des Américains possèdent ce statut. Par ailleurs, 13\% des Africains et des Asiatiques sont des requérants d'asile (permis $\mathrm{N}$ ), 11\% sont admis à titre provisoire (permis $\mathrm{F}$ ) et $7 \%$ ne disposent que d'une autorisation de séjour de courte durée (permis L). Deux tiers des Français et des Allemands possèdent un permis $\mathrm{C}$, le tiers restant se partageant entre ceux au bénéfice d'une autorisation de séjour annuelle (permis B) ou d'une autorisation de séjour de courte durée (voir graphique 3). On peut en conclure que la provenance et la filière d'immigration déterminent directement la durabilité du séjour en Suisse. Les accords bilatéraux, entrés en vigueur en 2002 comme mentionné plus haut, ont en outre sensiblement amélioré les conditions de séjour des ressortissants de l'Union européenne. Nombre de personnes qui vivaient sans autorisation en Suisse avant cette date (des Portugais par exemple) ont pu régulariser leur séjour. Les ressortissants non européens, notamment ceux au bénéfice d'une autorisation temporaire de séjour (soit 29298 personnes en 2007) ne possèdent que des droits restreints en matière de mobilité, d'intégration et de regroupement familial. Les

14 Rosita Fibbi, Mathias Lerch et Philippe Wanner, L'intégration des populations issues de l'immigration en Suisse: personnes naturalisés et deuxième génération, Neuchâtel, Office fédéral de la statistique, 2005, p. 57. 
perspectives de leur séjour en Suisse sont par conséquent incertaines. Ce constat vaut également pour les requérants d'asile (12243 personnes en 2007), qui n'ont qu'un accès limité au marché du travail pendant la procédure d'examen de leur demande.

\section{Graphique 3: Effectifs de la population résidante permanente étrangère, 1950-2007 (en milliers)}

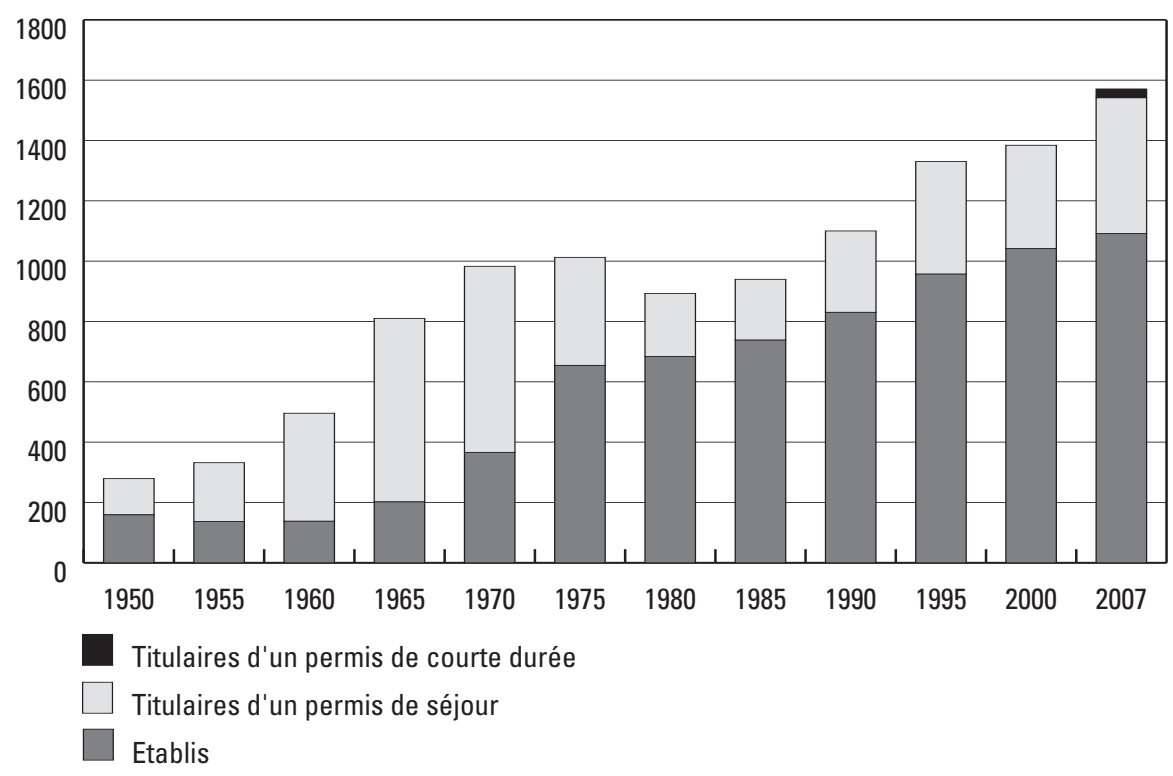

Source: Office fédéral des migrations (ODM), "Statistique des étrangers», 2007.

La mise en place réussie d'une approche duale de la politique d'admission et de la politique de l'emploi à la suite de la signature des accords sur la libre circulation des personnes avec l'Union européenne a marqué durablement la politique migratoire suisse. D'une part, les contingents et la priorité des travailleurs nationaux, qui ont caractérisé la politique d'immigration corporatiste qui visait à protéger les intérêts de certains secteurs et de certaines régions, appartiennent désormais au passé. D'autre part, il a aussi fallu relativiser, voire abandonner, l'approche sectorielle des problèmes migratoires, car la politique d'admission différenciée produisait des effets indésirables tant sur les futurs flux migratoires que sur leur gestion politique et administrative. Rappelons que cette politique avait été élaborée par l'administration dans les années 1990, afin de surmonter la «peur face à la surpopulation étrangère » et de permettre à la Suisse de se rapprocher de l'Union européenne. On était en effet parti de l'hypothèse que tant la main-d'œuvre européenne que les ressortissants non européens hautement qualifiés seraient mieux acceptés par la population.

Après la relégation, au début des années 1990, des régions traditionnelles de recrutement, comme l'ancienne Yougoslavie, du deuxième au troisième «cercle», le Conseil fédéral a en toute logique réagi à la détérioration de la situation des droits de l'homme peu avant l'éclatement des conflits dans les Balkans en fermant les filières saisonnières de recrutement. La volonté de faire 
preuve d'ouverture à l'égard de l'Europe tout en favorisant le recrutement de main-d'œuvre qualifiée en provenance de l'UE et de l'AELE a engendré, involontairement sans doute, une multiplication des demandes d'asile. Or, d'aucuns avaient pronostiqué une forte sollicitation de la filière de l'asile dès que la Yougoslavie a été exclue de la liste officielle des pays de recrutement.

L'administration a toutefois su tirer la leçon de ses erreurs. Au vu de la complexité du sujet, les arguments des partisans d'une politique migratoire globale, réunis autour de Peter Arbenz, ancien directeur de l'Office fédéral des réfugiés, eurent tôt fait de faire valoir leurs arguments. A une large majorité, le Parlement finit par demander au Conseil fédéral d'élaborer une loi sur les migrations, qui devait garantir un bilan d'émigration équilibré sur la base de critères tant humanitaires qu'économiques et politiques ${ }^{15}$. Les travaux devaient toutefois aussi tenir compte des exigences de la Commission des institutions politiques : la nouvelle loi sur les migrations devait fixer les objectifs et les limites de l'immigration, définir une politique globale d'intégration, créer les bases d'une promotion du retour au pays et contribuer à éliminer les causes des migrations. On jetait ainsi les fondements d'une stratégie migratoire globale à même de piloter, par le biais d'une coordination politique appropriée, les différents secteurs des politiques migratoire, d'asile et de développement. La prise en considération des divers aspects du phénomène migratoire s'est traduite par l'institution de groupes de travail interdépartementaux (IDAG Migration, par exemple), par l'appui à des initiatives internationales prenant la forme de partenariats sur les migrations (Initiative de Berne, Commission mondiale sur les migrations internationales, Dialogue de haut niveau sur les migrations internationales et le développement, Forum mondial sur la migration et le développement) et par l'adoption des nouvelles lois sur les étrangers (LEtr) et sur l'asile (LAsi), dont les textes reprennent des éléments du partenariat sur les migrations (voir art. 100 LEtr et art. 77 LAsi).

Par le passé, la nécessité de réunir les points de vue national et international s'est cependant souvent heurtée à des obstacles relevant de la politique intérieure. Il suffit de repenser au rejet massif de la politique migratoire par le peuple lors de votations organisées au cours des années 1990 pour se rendre compte du succès que doit remporter une approche holistique, en particulier lorsqu'elle concerne l'asile et l'immigration illégale. Voilà pourquoi nous nous intéressons ci-après plus particulièrement à ces deux formes du phénomène migratoire.

\section{L'asile}

Après 1945, la Suisse admit sa responsabilité dans le renvoi de réfugiés juifs à la frontière pendant la Seconde Guerre mondiale ${ }^{16}$. Désireux de s'amender et de réaffirmer sa volonté de préserver la tradition humanitaire du pays, le Conseil

15 Damir Skenderovic und Gianni D'Amato, Mit dem Fremden politisieren. Rechtspopulistische Parteien und Migrationspolitik in der Schweiz seit den 1960er Jahren, Zurich, Chronos, 2008, p. 157.

16 Voir Carl Ludwig, «La politique pratiquée par la Suisse à l'égard des réfugiés au cours des années 1933 à 1955: rapport adressé au Conseil fédéral à l'intention des conseils législatifs », in Carl Ludwig, La politique pratiquée par la Suisse à l'égard des réfugiés de 1933 à nos jours: annexe au rapport du Conseil fédéral à l'Assemblée fédérale [...], Berne, s.éd., 1957, pp. 1-359. 
fédéral a signé en 1955 la Convention de Genève, qui régissait depuis 1951 le statut du réfugié en droit international. Au cours des deux décennies qui suivirent, le pays pratiqua une politique libérale en matière d'asile et accueillit surtout avec générosité les ressortissants des pays communistes. A l'époque, la procédure était relativement simple et les réfugiés obtenaient généralement l'asile. C'est ainsi que 14000 Hongrois furent admis en 1956 et 12000 Tchécoslovaques en $1968^{17}$.

Ces réfugiés étaient souvent au bénéfice d'une bonne formation et reçurent un accueil des plus chaleureux, surtout en raison de l'anticommunisme qui prévalait à l'époque en Suisse. L'arrivée de quelques centaines de Chiliens, venus chercher refuge en Suisse après le putsch de Pinochet en 1973, provoqua une première controverse sur la légitimité du statut de réfugié qui leur a alors été accordé. Entre 1979 et 1982, la Suisse accorda encore, sur la base de contingents annuels, sa protection à 8000 boat people vietnamiens et cambodgiens. Leur intégration se révéla cependant plus difficile que celle des groupes de réfugiés venus précédemment ${ }^{18}$.

En 1981, la Suisse se dota de sa première loi sur l'asile, qui réglementait la pratique généreuse appliquée jusqu'alors. La loi définissait les règles régissant l'octroi du statut de réfugié, fixait la procédure et donnait à la Confédération un moyen d'intervention politique. Son application incombait cependant aux cantons. Ceux-ci ne disposant pas tous des mêmes capacités en matière de prévoyance sociale, de perfectionnement professionnel et de rapatriement, la mise en œuvre de la loi variait d'un canton à l'autre ${ }^{19}$.

Après 1981, deux tendances se dessinèrent: le nombre des demandes, qui avait doublé au cours des années 1970 pour atteindre 1000 par an, suivit une hausse exponentielle. De plus, la plupart des requérants (à l'exception des Polonais arrivés en 1982) venaient du «Sud», en majorité de régions ou de pays tels que la Turquie, le Sri Lanka, le Moyen-Orient, l'Afrique ou l'Asie. Contrairement aux réfugiés qui avaient fui le communisme, ces requérants ne possédaient pas toujours de bonnes qualifications ou des diplômes professionnels suffisants. Dès le milieu des années 1980, la politique d'asile devint un sujet politiquement sensible, car cette forme de migration est protégée par le droit humanitaire international. Lors de débats publics, d'aucuns ont associé une connotation négative à la notion de «demandeur d'asile», afin de bien faire sentir que les personnes venues chercher refuge en Suisse n'étaient pas mues par des raisons légitimes, qu'elles usurpaient le statut de réfugié et que leur seul objectif était d'obtenir un permis de séjour. Très présent dans l'opinion publique, ce discours a conduit les

17 Denise Efionayi-Mäder et al., «Switzerland», in Jan Niessen and Yongmi Schibel (eds.), EU and US Approaches to the Management of Immigration: Comparative Perspectives, Brussels, Migration Policy Group, 2003, pp. 491-519.

18 Lorena Parini et Matteo Gianni, La tension entre précarité et intégration: politique à l'égard des migrants en Suisse, Genève, Département de science politique de l’Université de Genève, 1996, 21 p. Lorena Parini et Matteo Gianni, «Enjeux et modifications de la politique d'asile en Suisse de 1956 à nos jours », in Hans Mahnig (dir.), Histoire de la politique de migration, d'asile et d'intégration en Suisse depuis 1948, Zurich, Seismo, 2005, pp. 189-252.

19 Thomas Holzer et Gerald Schneider, «Le revers de la médaille d'une mise en œuvre décentralisée des procédures d'asile : à l'exemple de la Suisse, perspectives pour l'Union européenne », in Hans-Rudolf Wicker, Rosita Fibbi et Werner Haug (dir.), Les migrations et la Suisse: Résultats du Programme national de recherche «Migrations et relations interculturelles», Zurich, Seismo, 2003, pp. 153-174. 
autorités à revoir à plusieurs reprises la Loi sur l'asile pour l'adapter à des besoins prétendument politiques, à interpréter par conséquent la procédure d'asile plus strictement et à accepter moins de requérants, même s'ils provenaient de régions en guerre. L'évolution du taux d'admission témoigne de cette tendance: de $86 \%$ entre 1975 et 1979, il est passé à $46 \%$ entre 1980 et 1984, puis à $6 \%$ entre 1985 et $1990^{20}$.

L'asile occupa une place toujours plus grande dans le débat public, au point d'éclipser la question naguère controversée des travailleurs migrants dans l'actualité politique. Si les années 1990 furent marquées par un taux de reconnaissance très bas, beaucoup de requérants d'asile furent néanmoins admis en Suisse pour des raisons humanitaires. Et, bien que les cantons eussent le plus souvent restreint leurs droits pendant la première phase de leur séjour (accès limité au marché du travail et aux prestations sociales, refus du regroupement familial), les requérants dont le droit à une protection fut reconnu purent ensuite s'établir durablement en Suisse. A cette période, ce furent principalement les guerres des Balkans qui engendrèrent de nouveaux afflux de réfugiés qui fuyaient la Bosnie et le Kosovo. Certains d'entre eux avaient des proches en Suisse, qui étaient venus y chercher du travail avant la guerre civile. Entre 1990 et 2002, 146587 personnes issues des régions en guerre dans les Balkans ont demandé l'asile en Suisse ${ }^{21}$.

Dans les années 1990, l'opinion publique a à nouveau manifesté sa mauvaise humeur face à l'accroissement du nombre de demandes d'asile, surtout parce que le chômage dû à la crise structurelle évoquée plus haut frappait durement l'économie suisse. Le Conseil fédéral adopta dès lors de nouvelles mesures administratives et légales pour accélérer la procédure d'asile et mettre en œuvre les décisions prises à son issue. La Loi sur l'asile resta en chantier et fut à nouveau révisée en 1999. Cette révision introduisit de nouvelles exigences à même d'empêcher les requérants d'asile d'accéder à la procédure. Les dispositions en question visaient en particulier à empêcher les requérants présents illégalement dans le pays et n'étant pas en mesure de présenter des papiers d'identité de séjourner longtemps en Suisse. A titre de concession aux réserves émises par les œuvres d'entraide, la nouvelle loi réglementait l'admission collective pour une durée limitée des réfugiés de guerre, une réglementation dont les Kosovars et les Bosniaques furent les principaux bénéficiaires. Après les guerres des Balkans, environ 60000 personnes retournèrent dans leur pays d'origine, tandis que quelque 10000 réfugiés de guerre s'établirent définitivement en Suisse. Le nombre de ceux qui y vivent tant bien que mal dans l'illégalité n'est pas connu ${ }^{22}$.

Bien que le climat politique qui a régné pendant les campagnes menées lors de la révision de la Loi sur l'asile au cours des années 1980 et 1990 rappelât le

20 Denise Efionayi-Mäder, «Asylpolitik der Schweiz 1950-2000», Asyl, n² 2, 2003, pp. 3-9.

Ce taux augmenta à nouveau légrèement (9\%) à partir du milieu des années 1990 et se stabilisa jusqu'en 2004. Dès 2005, le taux d'admission s'accrut sensiblement pour attindre $18 \%$ (voir <http://www.bfm.admin.ch/etc/medialib/data/migration/statistik/asylstatistik/uebersichten.Par.0003. File.tmp/Asyl-Uebersicht-Jahre-Total-d.pdf $>$ ).

21 Bülent Kaya, «Switzerland», in Jan Niessen, Yongmi Schibel and Cressida Thompson (eds.), Current Immigration Debates in Europe: A Publication of the European Migration Dialogue, Brussels, Migration Policy Group, 2005, pp. 383-398.

22 Denise Efionayi, Josef Martin Niederberger and Philippe Wanner, «Switzerland Faces Common European Challenges », Migration Information Source (MPI), February 2005. 
discours xénophobe qui avait auparavant marqué les votations sur les étrangers, il ne faut pas oublier que les requérants d'asile trouvaient du travail exactement dans les mêmes secteurs qui avaient naguère employé les saisonniers. Si nul ne les appelait expressément main-d'œuvre étrangère, les réfugiés au bénéfice de l'asile ont contribué au développement économique. Etienne Piguet et Andreas Wimmer furent les premiers à émettre l'hypothèse que les requérants d'asile étaient les nouveaux Gastarbeiter (travailleurs étrangers) en Suisse ${ }^{23}$, hypothèse qui fut confirmée par un travail de recherche de Jürg Kuster et Guido Cavelti ${ }^{24}$. Relevons que si les requérantes et requérants d'asile travaillent dans les mêmes branches que les saisonniers, c'est surtout en raison des mesures appliquées par les autorités qui régulent l'accès au marché du travail. Comme les ressortissants de pays non membres de l'UE ne peuvent plus, depuis les années 1990, obtenir un permis saisonnier, mais que les besoins du marché du travail en maind'œuvre non qualifiée doivent néanmoins être couverts, l'économie s'est en désespoir de cause tournée vers les requérants d'asile ${ }^{25}$. Ceux-ci offrent en effet aux marchés périphériques du travail une flexibilité optimale, dont profitent surtout l'hôtellerie, la construction et le secteur du nettoyage ${ }^{26}$. La situation s'explique par l'assèchement du marché du travail, même dans le domaine des emplois peu qualifiés. En marge de l'arrivée d'une main-d'œuvre européenne hautement qualifiée, expressément souhaitée par les autorités, une autre immigration intervient ainsi depuis les années 1990, qui s'affirme de plus en plus sur le plan économique bien qu'elle demeure indésirable sur le plan rhétorique et symbolique, car sa motivation paraît moins respectable (fuite devant la misère et fuite des zones en conflit). La connotation symbolique associée à cette migration due à la misère a des conséquences sur la perception politique et sociale de formes indésirables de l'immigration, notamment l'immigration illégale.

\section{L'immigration illégale}

En réaction à l'augmentation des demandes d'asile dans les années 1980 et à l'avis général que la majorité des requérants étaient de «faux réfugiés», toute une série de politiques furent mises en œuvre au cours de la décennie suivante. Elles visaient à durcir le droit d'asile afin d'abaisser le nombre des requérants et de couper l'herbe sous les pieds des partis de la droite populiste, qui s'obstinaient à faire de l'asile leur cheval de bataille. La Suisse a ainsi introduit nombre de mesures restrictives dans l'aide sociale, l'accès au marché du travail et d'autres domaines (formation, soins de santé, mobilité, etc.), qui ne s'appliquent qu'aux requérants d'asile dont elles limitent considérablement la mobilité sociale et spatiale. Au niveau européen, les Accords de Schengen de 1985 et la

23 Etienne Piguet et Andreas Wimmer, «Les nouveaux Gastarbeiter? Les réfugiés sur le marché du travail suisse», Revue de l'intégration et de la migration internationale, vol. 1, nº 2, 2002, pp. 233-257.

24 Jürg Kuster et Guido Cavelti, «Le recrutement de la main-d'œuvre étrangère: effets des règlements du droit des étrangers et du droit d'asile», in Hans-Rudolf Wicker, Rosita Fibbi et Werner Haug (dir.), Les migrations et la Suisse: résultats du Programme national de recherche "Migrations et relations interculturelles », Zurich, Seismo, 2003, pp. 247-262.

25 Rosita Fibbi et Janine Dahinden, «Les requérants d'asile et le travail: déclassés? indispensables? bienvenus?», Asyl, vol. 19, n 3, 2004, p. 21.

26 Etienne Piguet, Stefano Losa et Jean-Hugues Ravel, «Demandeurs d'asile et travailleurs étrangers clandestins sur le marché du travail suisse», Asyl, vol. 17, n² 2, 2002, pp. 3-8. 
Convention de Dublin de 1990 ont mis en place un régime migratoire communautaire qui, d'une part, abolit les contrôles douaniers au sein de l'UE en créant un espace unique où les citoyens de l'UE sont libres d'aller et venir à leur guise, mais qui, d'autre part, renforce les exigences en matière de visa pour les ressortissants de toute une série de pays tiers, rendant impossible, malgré les intentions officiellement affichées, une immigration réglementée à parti de ces pays $^{27}$. Depuis 2008, ces accords s'appliquent également en Suisse. La désignation d' «Etats sûrs », dans lesquels la Suisse peut renvoyer des requérants d'asile sans enfreindre le principe du non-refoulement inscrit dans la Convention de Genève, a créé une zone tampon autour de l'Union européenne, qui englobait les anciens pays communistes d'Europe centrale et d'Europe de l'Est. Après leur intégration dans l'Union européenne, cette zone tampon fut repoussée plus à l'Est et plus au Sud. Pour éviter les demandes d'asile multiples, les décisions rendues en matière d'asile par l'un des Etats membres sont valables dans tous les Etats signataires des accords. Un requérant ne peut donc demander l'asile que dans un seul pays. Des accords bilatéraux de réadmission permettent de renvoyer un requérant d'asile débouté dans l'un des Etats tiers sûrs par lesquels il a transité. Les nouvelles réglementations ont non seulement introduit la possibilité de déposer la demande d'asile dans le pays de provenance (in-coutry processing) et de condamner l'entreprise de transport aérien ayant accepté de prendre en charge des passagers ne possédant pas une autorisation d'entrée dans le pays (carrier sanctions), mais ont aussi tenté de canaliser les flux migratoires dans d'autres directions. Les Etats de l'Union européenne ont par ailleurs déplacé les contrôles d'immigration vers les pays de provenance et imposé leur responsabilité aux entreprises de transport aérien en exigeant des contrôles avant embarquement (pre-boarding checks), d'où une privatisation de facto des contrôles aux frontières (remote control).

La combinaison des diverses réglementations a eu pour effet de restreindre toujours plus l'interprétation de la notion de réfugiée selon l'article 1 de la Convention de Genève et de réduire de manière impressionnante le nombre des requérants d'asile en Europe occidentale après le pic atteint en 1992. Après un succès initial, l'impact des nouvelles mesures s'estompa cependant vers la fin des années 1990. Le nouvel accroissement du nombre de demandes d'asile enregistrées alors s'explique par le fait que nombre de migrants et de requérants eurent recours, après la fermeture des «canaux officiels de l'asile», à d'autres filières, irrégulières celles-là, pour passer les frontières, tout comme la clôture des programmes de recrutement de travailleurs étrangers avait incité nombre de candidats à l'émigration à emprunter la «voie de l'asile». Divers indices montrent par ailleurs que ce sont surtout les passeurs qui tendent à monopoliser les filières illégales d'entrée dans le pays ${ }^{28}$. Reste cependant à savoir ce que désigne la notion d' «immigration illégale».

L'immigration illégale, au sens où nous l'entendons ici, désigne tous les phénomènes liés à l'entrée illicite et au séjour non autorisé de ressortissants étrangers

27 Sandra Lavenex and Emek M. Uçarer (eds.), Migration and the Externalities of European Integration, Lanham (etc.), Lexington Books, 2002.

28 Khalid Koser, «The Smuggling of Asylum Seekers into Western Europe: Contradictions, Conundrums, and Dilemmas», in David Kyle and Rey Koslowski (eds.), Global Human Smuggling: Comparative Perspectives, Baltimore, MD, Johns Hopkins University Press, 2001. 
dans un pays. On parle aussi souvent d' «immigration clandestine», d' «étrangers en situation irrégulière», de «sans-papiers», etc. La variété des expressions utilisées tend à rappeler que l'illégalité relève ici d'une question de perspective ${ }^{29}$. Voici quelques-unes des formes qu'elle peut adopter:

$1^{\circ}$ passage illicite (en fraude) de la frontière et séjour clandestin (situation classique des sans-papiers);

$2^{\circ}$ passage illicite et séjour autorisé (requérants d'asile par exemple);

$3^{\circ}$ entrée licite dans le pays et dépassement de la durée autorisée du séjour, qui aboutit à un séjour illégal ou contraire aux conditions de visa (c'est la situation de ce que l'on appelle les overstayers).

L'expression «sans-papiers», qui désigne des personnes sans autorisation de séjour valable, a été créée en France dans les années 1970 pour remplacer des termes très méprisants qui étaient associés à la délinquance («illégaux», «clandestins»). La littérature anglaise spécialisée distingue souvent entre sanspapiers (undocumented migrant), entrés illégalement dans le pays mais au bénéfice d'un droit de séjour, et immigrés entrés légalement mais dont le droit de séjour est échu (overstayers). Cette dernière situation survient par exemple à la suite du non-renouvellement de l'autorisation de séjour, de l'expiration du visa de tourisme, de l'achèvement des études, du refus de l'asile, de la perte de l'emploi, d'un divorce ou du décès du conjoint, ou dans le cas de personnes tributaires de l'assistance sociale qui se sont rendues coupables d'un délit ou qui sont parvenues au terme d'un bref séjour autorisé.

La situation juridique des personnes sans autorisation de séjour est extrêmement complexe: à l'instar des immigrés dûment enregistrés, ces personnes bénéficient des droits humains ainsi que de certains droits sociaux et du travail, mais ne peuvent les faire valoir qu'au risque d'être expulsées du pays. Bien que l'on considère en général que les sans-papiers sont responsables de leur situation illégale, il ne faut pas oublier que nombre d'entreprises et de ménages profitent de cette main-d'œuvre, en général très bon marché. Par ailleurs, le durcissement incessant de la réglementation sur les étrangers et sur l'asile a certainement contribué à grossir les rangs des ressortissants étrangers en situation illégale. Les causes de l'immigration illégale sont cependant aussi diverses et variés que les conditions et les parcours de vie des personnes sans statut de séjour. L'expérience a ainsi montré qu'il est impossible d'éviter les mouvements migratoires illégaux, même lorsque des perspectives d'immigration légale existent bel et bien. On n'est sûr que d'une chose: seule la libre circulation des personnes pourra venir à bout de ce phénomène.

Même si les informations sur les sans-papiers sont rares, les indications fournies par les professionnels et les données partielles disponibles permettent de brosser leur portrait ${ }^{30}$. En Suisse, il s'agit principalement de personnes venues d'Amérique latine (surtout des femmes), des Etats issus de l'ancienne Yougoslavie, d'Europe de l'Est, de Turquie, ainsi que de quelques pays d'Afrique et d'Asie.

29 Bimal Ghosh, Huddled Masses and Uncertain Shores: Insights into Irregular Migration, The Hague (etc.), Martinus Nijhoff Publishers, 1998, p. 1.

30 Denise Efionayi-Mäder et Christin Achermann, «Sans papiers, mais pas sans droits», Sécurité sociale, $\mathrm{n}^{\circ} 6,2003$, p. 359. 
La majorité d'entre elles ont entre 20 et 40 ans et si beaucoup de femmes vivent dans les villes, on trouve aussi des familles et des pères élevant seuls leurs enfants en situation illégale. Les sans-papiers sont au bénéfice d'une formation relativement bonne, mais leurs salaires sont souvent plus bas que ceux de la main-d'œuvre immigrée légale. La grande majorité d'entre eux exercent une activité lucrative, en particulier dans les travaux ménagers (nettoyage, garde des enfants, soins aux personnes âgées, etc.), le secteur du nettoyage, l'hôtellerie et la restauration, la construction, l'agriculture et l'industrie du sexe.

Jusqu'à la fin des années 1990, l'illégalité est demeurée un sujet marginal en Suisse alémanique. Confinant au tabou, elle n'était ouvertement mise sur la table que par les défenseurs des sans-papiers. Des recherches ont cependant contribué à faire valoir qu'il n'est guère possible de compter sur des réglementations nationales ou internationales pour piloter l'immigration dans un monde fait d'interdépendances. Entre les limites imposées par les Etats nationaux et la réalité socio-économique, autorités et organisations non gouvernementales doivent dès à présent trouver au problème des sans-papiers des solutions qui ne peuvent que s'écarter d'une application stricte de la loi. Il reste encore à élaborer une politique dans ce domaine qui d'une part respecte les droits humains et, d'autre part, propose des approches crédibles du phénomène migratoire.

\section{Conclusions}

Une approche sectorielle axée sur un seul aspect de la politique, ne permet donc pas d'appréhender l'évolution des migrations à laquelle la Suisse et d'autres pays occidentaux ont été confrontés ces dernières années. Le «meilleur» traitement réservé à l'immigration en provenance du sud de l'Europe, que la Suisse a connue dans les années 1950 et 1960, surprend sans doute lorsque l'on considère la «nouvelle» immigration des années 1980 et 1990. Les «privilèges» accordés à l'immigration européenne sont en grande partie à mettre sur le compte de la forte demande de main-d'œuvre de l'époque, qui fut satisfaite dans le cadre d'accords bilatéraux conclus avec les pays d'origine. La mise en place de mécanismes de régulation n'a cependant pas suffi pour prévenir le mécontentement politique, comme en témoignent les critiques incessantes de la part des mouvements populistes au sujet de la «surpopulation étrangère». Le Conseil fédéral pouvait néanmoins, quoique l'entreprise n'eût rien d'aisé, contrer ces critiques, car il s'agissait en fin de compte d'une immigration voulue de maind'œuvre $^{31}$. La venue de réfugiés du Sud dès le milieu des années 1980, les guerres qui secouèrent l'Europe du Sud-Est dans les années 1990 et l'ampleur, volontairement grossie, de l'immigration illégale ont marqué un tournant qui a sapé une répartition de la politique migratoire en secteurs clairement distincts: d'une part, les territoires de l'ancienne Yougoslavie ont été exclus des régions de recrutement au début des années 1990; d'autre part, le Conseil fédéral a souligné la nécessité de concevoir à l'avenir une politique migratoire globale ${ }^{32}$.

31 Damir Skenderovic und Gianni D'Amato, op. cit.

32 Peter Arbenz, Rapport sur une politique suisse en matière de migrations: avant-projet du 25.3.1994, Berne, Département fédéral de justice et police (DFJP), 1994. Peter Arbenz, Rapport sur une politique suisse en matière de migrations, s.l., s.éd., 1995. Peter Arbenz, «De la politique d'asile à une politique migratoire», in Philippe Henry, Gaston Gaudard et Peter Arbenz (dir.), La Suisse terre 
Depuis lors, les points communs entre politiques du travail, d'asile et de développement n'ont cessé de se renforcer. Ce renforcement transparaît notamment dans le cas du lien entre la politique des renvois et les aides structurelles fournies aux anciennes régions de provenance et dans les efforts du Conseil fédéral pour prévenir l'immigration illégale ${ }^{33}$.

Ces dernières années ont été marquées par les efforts de la Suisse et d'autres gouvernements occidentaux pour conclure des partenariats avec des membres des autorités, des politiciens, des membres de la société civile et des scientifiques du Sud, afin d'aborder les problèmes actuels au sein de forums internationaux ${ }^{34}$. Cette politique reflète cependant, elle aussi, l'opinion dominante selon laquelle les migrations sont un problème qu'il convient d'aborder et de régler au moyen de politiques appropriées. Tandis que les partisans de solutions répressives avancent les contrôles aux frontières et la préservation de la souveraineté politique et territoriale, les tenants d'une politique libérale souhaitent s'attaquer aux causes des migrations (le plus souvent la pauvreté et la violence), pour que les gens ne soient plus obligés d'émigrer. Les migrations sont donc considérées comme un dysfonctionnement, auquel il faut remédier. On retrouve ici un point commun entre les anciennes administrations coloniales et les organismes modernes de coopération au développement, qui ont de tout temps préféré voir les travailleurs migrants rester chez eux. Les pays riches et industrialisés ayant toujours besoin de main-d'œuvre étrangère, les recommandations émises lors de ces rencontres ne conduisent pas à une interdiction absolue de l'immigration de travailleuses et de travailleurs peu qualifiés, mais souvent à la diffusion de l'idée que des «migrations circulaires » pourraient profiter à tout le monde, tant aux pays d'origine et de destination qu'aux migrants eux-mêmes. Au terme du «processus de circulation », les migrants retournent bien entendu chez eux... ${ }^{35}$

Les migrations constituent de toute évidence un aspect des temps modernes que les analyses actuelles n'associent pas assez aux profonds changements qui caractérisent notre époque. Or, ce sont justement ces changements universels qui expliquent la complexité, l'intrication, la versatilité et les traits contextuels d'un phénomène (la migration) qui intervient à plusieurs niveaux et trouve son origine dans la répartition inéquitable des richesses à l'échelle mondiale. Malgré sa justesse, cette explication structurelle ne signifie pas que les flux migratoires seraient moindres dans un monde où le développement serait plus égalitaire. On assisterait plutôt à d'autres types de migrations. Les facteurs économiques n'expliquent donc qu'une partie du phénomène et ne suffisent pas pour décrire toute la complexité subtile du phénomène migratoire dans une civilisation en profonde mutation.

Pour le comprendre, nous devrions nous fonder sur une notion du changement qui parte des sociétés occidentales postindustrielles et tienne compte des restruc-

d'asile, Bienne, Editions Libertas Suisse, 1995, pp. 65-81. Office fédéral de l'industrie, des arts et métiers et du travail (OFIAMT) et Office fédéral des étrangers (OFE), Rapport sur la conception et les priorités de la politique suisse des étrangers pour les années 1990, Berne, Office central des imprimés et du matériel, 1991.

33 Projektteam PiM, Abschlussbericht Prävention irregulärer Migration, Berne, s.éd., 2003.

34 Voir la contribution de Pietro Mona (DFAE) dans le présent volume.

35 Stephen Castles, «Understanding Global Migration: A Social Transformation Perspective », paper for the IMISCOE Conference on Theories of Migration and Social Change, St Anne's College, University of Oxford, 1-3 July 2008. 
turations mondiales, au niveau tant des investissements que de la production et du commerce de biens et de connaissances. Dans les pays industrialisés, c'est surtout après la crise de 1973 que l'on a assisté à la disparition d'anciennes industries, à la restructuration du marché de l'emploi, à l'érosion de l'Etat-providence et à la dissolution des milieux prolétaires jusqu'alors efficacement organisés. Ces processus de restructuration ont fini par favoriser les migrations à la fois de main-d'œuvre hautement qualifiée et de main-d'œuvre non qualifiée. Dans les pays moins développés, cette transformation amorcée dans l'hémisphère Nord du globe a conduit à la rationalisation et à la destruction de modes de vie ruraux, à l'érosion de l'ordre social, à l'exode rural et à l'apparition de bidonvilles dans les métropoles - autant de changements qui tous favorisent les migrations.

Les processus observés sont finalement à mettre sur le compte d'une nouvelle division des marchés internationaux du travail. Aux postes de travail garantis dans les grands secteurs de production correspondent des emplois précaires sur les marchés périphériques du travail. La possibilité pour une personne de trouver un emploi sur les marchés privilégiés ne dépend pas seulement de son capital humain, c'est-à-dire de ses connaissances et de ses compétences, mais aussi de son sexe, de son origine et de son statut légal. Le parcours de chaque migrant résulte ainsi d'une combinaison complexe entre l'évolution des marchés de l'emploi et les grands processus de changement (post)industriels au Sud et au Nord.

Pour élaborer une politique capable d'aborder le phénomène migratoire de manière productive, il importe dès lors de garder deux choses à l'esprit: premièrement, les migrations font partie de l'histoire de l'humanité et, deuxièmement, la phase actuelle de changement, dont les effets se font sentir plus ou moins durement partout dans le monde, a créé un nouveau type de migrations. Si l'on veut influer sur ce phénomène, il importe d'en saisir toute la complexité et toute l'envergure, afin de le décliner ensuite de manière réaliste.

A l'heure actuelle, il n'existe pas de théorie globale de ce type, tout comme il n'existe pas de politique migratoire globale, c'est-à-dire un code de conduite appliqué par une majorité d'Etats. Les intérêts des gouvernements sont en effet trop divergents pour que ceux-ci parviennent à s'entendre sur une gestion commune des migrations. Dans ce domaine, seule l'Union européenne a réussi à établir un consensus au niveau régional. Le prix en est toutefois que l'on considère les migrations internes comme une mobilité voulue de main-d'œuvre hautement qualifiée, tandis que les déplacements de la main-d'œuvre peu qualifiée en provenance de pays non européens constituent des migrations non désirées. La mobilité est un bien souhaitable, car elle est le fait de sociétés ouvertes et autodéterminées, tandis que les migrations portent en elles le poids de la contrainte et sont généralement associées à des communautés archaïques. Face à une telle controverse quant à l'interprétation du phénomène migratoire, la recherche se doit de dépasser les besoins de la politique quotidienne pour considérer les divers aspects, parfois contradictoires, du monde moderne. C'est là que réside sa chance de parvenir à expliquer les migrations - et son propre rôle. 


\section{Bibliographie}

Arbenz, Peter, «De la politique d'asile à une politique migratoire», in Philippe Henry, Gaston Gaudard et Peter Arbenz (dir.), La Suisse terre d'asile, Bienne, Editions Libertas Suisse, 1995, pp. 65-81.

Arbenz, Peter, Rapport sur une politique suisse en matière de migrations: avant-projet du 25.3.1994, Berne, Département fédéral de justice et police (DFJP), 1994.

Arbenz, Peter, Rapport sur une politique suisse en matière de migrations, s.l., s.éd., 1995.

Bolzman, Claudio, Rosita Fibbi et Marie Vial, Secondas - secondos: le processus d'intégration des jeunes adultes issus de la migration espagnole et italienne en Suisse, Zurich, Seismo, 2003.

Busset, Thomas, «La politique du refuge en Suisse 1820-1870. Réalité et mythe», Zeitschrift des Schweizerischen Bundesarchivs, $\mathrm{n}^{\circ}$ 25, 1999, pp. 29-64.

Castles, Stephen, «Understanding Global Migration: A Social Transformation Perspective», paper for the IMISCOE Conference on Theories of Migration and Social Change, St Anne's College, University of Oxford, 1-3 July 2008.

Cerutti, Mauro, «L'immigration italienne en Suisse dans le contexte de la Guerre froide», in Jean Batou, Mauro Cerutti et Charles Heimberg (dir.), Pour une histoire des gens sans histoire: ouvriers, exclus et rebelles en Suisse: XIX ${ }^{e}-X X^{e}$ siècles, Lausanne, Editions d'En Bas, 1995, pp. 213-231.

D’Amato, Gianni und Brigitta Gerber (Hrsg.), Herausforderung Integration: städtische Migrationspolitik in der Schweiz und in Europa, Zurich, Seismo, 2005.

Davis, Kingsley, «Social Science Approaches to International Migration», Population and Development Review, vol. 14, n 4, 1988, pp. 245-261.

Efionayi, Denise, Josef Martin Niederberger and Philippe Wanner, «Switzerland Faces Common European Challenges », Migration Information Source (MPI), February 2005.

Efionayi-Mäder, Denise, «Asylpolitik der Schweiz 1950-2000», Asyl, n 2, 2003, pp. 3-9.

Efionayi-Mäder, Denise et Christin Achermann, «Sans papiers, mais pas sans droits», Sécurité sociale, $n^{\circ} 6,2003$, p. 359.

Efionayi-Mäder, Denise et al., «Switzerland», in Jan Niessen and Yongmi Schibel (eds.), EU and US Approaches to the Management of Immigration: Comparative Perspectives, Brussels, Migration Policy Group, 2003, pp. 491-519.

Fibbi, Rosita et Janine Dahinden, «Les requérants d'asile et le travail: déclassés ? indispensables ? bienvenus?», Asyl, vol. 19, n 3, 2004, pp. 21-27.

Fibbi, Rosita, Mathias Lerch et Philippe Wanner, L'intégration des populations issues de l'immigration en Suisse: personnes naturalisés et deuxième génération, Neuchâtel, Office fédéral de la statistique, 2005.

Fragnière, Jean-Pierre et Roger Girod (dir.), Dictionnaire suisse de politique sociale, Lausanne, Réalités sociales, 2002.

Freiburghaus, Dieter und Brigitte Guggisberg, «Die schweizerische Ausländerpolitik seit 1850 : eine Analyse auf dem Hintergrund politisch-ökonomischer Paradigmen », in Thomas Geiser, Hans Schmid und Emil Walter-Busch (Hrsg.), Arbeit in der Schweiz des 20. Jahrhunderts: wirtschaftliche, rechtliche und soziale Perspektiven, Bern (etc.), P. Haupt, 1998, pp. 137-185.

Ghosh, Bimal, Huddled Masses and Uncertain Shores: Insights into Irregular Migration, The Hague (etc.), Martinus Nijhoff Publishers, 1998.

Holmes, Madelyn, Forgotten Migrants: Foreign Workers in Switzerland before World War I, Rutherford (etc.), Fairleigh Dickinson University Press (etc.), 1998.

Holzer, Thomas et Gerald Schneider, «Le revers de la médaille d'une mise en œuvre décentralisée des procédures d'asile: à l'exemple de la Suisse, perspectives pour l'Union européenne», in Hans-Rudolf Wicker, Rosita Fibbi et Werner Haug (dir.), Les migrations et la Suisse: résultats du Programme national de recherche "Migrations et relations interculturelles », Zurich, Seismo, 2003, pp. 153-174.

Kaya, Bülent, «Switzerland», in Jan Niessen, Yongmi Schibel and Cressida Thompson (eds.), Current Immigration Debates in Europe: A Publication of the European Migration Dialogue, Bruxelles, Migration Policy Group, 2005, pp. 383-398.

Koser, Khalid, «The Smuggling of Asylum Seekers into Western Europe: Contradictions, Conundrums, and Dilemmas », in David Kyle and Rey Koslowski (eds.), Global Human Smuggling: Comparative Perspectives, Baltimore, MD, Johns Hopkins University Press, 2001.

Kuster, Jürg et Guido Cavelti, «Le recrutement de la main-d'œuvre étrangère: effets des règlements du droit des étrangers et du droit d'asile», in Hans-Rudolf Wicker, Rosita Fibbi et Werner Haug (dir.), Les migrations et la Suisse: résultats du Programme national de recherche "Migrations et relations interculturelles», Zurich, Seismo, 2003, pp. 247-262.

Lavenex, Sandra et Emek M. Uçarer (eds.), Migration and the Externalities of European Integration, Lanham (etc.), Lexington Books, 2002. 
Ludwig, Carl, «La politique pratiquée par la Suisse à l'égard des réfugiés au cours des années 1933 à 1955 : rapport adressé au Conseil fédéral à l'intention des conseils législatifs », in Carl Ludwig, La politique pratiquée par la Suisse à l'égard des réfugiés de 1933 à nos jours: annexe au rapport du Conseil fédéral à l'Assemblée fédérale [...], Berne, s.éd., 1957, pp. 1-359.

Mahnig, Hans et Etienne Piguet, «La politique d'immigration suisse de 1948 à 1998: évolution et effets », in Hans-Rudolf Wicker, Rosita Fibbi et Werner Haug (dir.), Les migrations et la politique suisse: résultats du Programme national «Migrations et relations interculturelles », Zurich, Seismo, 2003, pp. 63-103.

Office fédéral de l'industrie, des arts et métiers et du travail (OFIAMT) et Office fédéral des étrangers (OFE), Rapport sur la conception et les priorités de la politique suisse des étrangers pour les années 1990, Berne, Office central des imprimés et du matériel, 1991.

Parini, Lorena et Matteo Gianni, «Enjeux et modifications de la politique d'asile en Suisse de 1956 à nos jours », in Hans Mahnig (dir.), Histoire de la politique de migration, d'asile et d'intégration en Suisse depuis 1948, Zurich, Seismo, 2005, pp. 189-252.

Parini, Lorena et Matteo Gianni, La tension entre précarité et intégration: politique à l'égard des migrants en Suisse, Genève, Département de science politique de l'Université de Genève, 1996, 21 p.

Piguet, Etienne, Einwanderungsland Schweiz: fünf Jahrzehnte halb geöffnete Grenzen, Berne [etc.], Haupt Verlag, 2006.

Piguet, Etienne et Andreas Wimmer, «Les nouveaux Gastarbeiter? Les réfugiés sur le marché du travail suisse», Revue de l'intégration et de la migration internationale, vol. 1, no 2, 2002, pp. 233-257.

Piguet, Etienne, Stefano Losa et Jean-Hugues Ravel, «Demandeurs d'asile et travailleurs étrangers clandestins sur le marché du travail suisse», Asyl, vol. 17, n 2, 2002, pp. 3-8.

Projektteam PiM, Abschlussbericht Prävention irregulärer Migration, Berne, s.éd., 2003.

Skenderovic, Damir und Gianni D’Amato, Mit dem Fremden politisieren. Rechtspopulistische Parteien und Migrationspolitik in der Schweiz seit den 1960er Jahren, Zurich, Chronos, 2008.

Urner, Klaus, Die Deutschen in der Schweiz: von den Anfängen der Kolonienbildung bis zum Ausbruch des Ersten Weltkrieges, Frauenfeld (etc.), Huber, 1976.

Vuilleumier, Marc, «L'emigrazione italiana in Svizzera e gli avvenimenti del 1898 », in AA. VV., Anna Kuliscioff e l'età del riformismo: atti del convegno di Milano, dicembre 1976, Roma, Mondo operaio, 1978, pp. 85-103.

Vuilleumier, Marc, «Le mouvement ouvrier et les travailleurs italiens en Suisse jusqu'à la Première Guerre mondiale», Revue syndicale suisse, vol. 82, n 4, 1990, pp. 102-116.

Wanner, Philippe, Migration et intégration: population étrangère en Suisse, Neuchâtel, Office fédéral de la statistique, 2004. 